\title{
Theoretical Study on Indacaterol by DFT Study
}

\author{
J. Winfred Jebaraj ${ }^{1}$, P. Muthuselvan ${ }^{1}$, D. Jim Livingston ${ }^{1}$ I. Antony Danish ${ }^{2}$ \\ ${ }^{I}$ Department of Chemistry, St. John's College, Palayamkottai, India - 627002 \\ ${ }^{2}$ Department of Chemistry, Sadakathullah Appa College, Palayamkottai, India - 627011
}

\begin{abstract}
Quantum chemical calculations were carried out to study the molecular structure for indacaterol. To investigate the optimized molecular structure, bond length, bond angle and tetrahedral angels, Mullikan atomic charges, HOMO, LUMO energy levels, energy gap, dipole moment, total energy and some other physical parameters, DFT calculations were carried out using 6-31G basis set with B3LYP.
\end{abstract}

Keywords: Dipole moment, HOMO-LUMO energy gap, Indacaterol, Mullikan charges.

\section{Introduction}

Indacaterol is a drug used for the treatment of chronic obstructive pulmonary disease (COPD). It's chemical name is 5-\{(1R)-2-[(5,6-diethyl-2,3-dihydro-1H-inden-2-yl)amino]-1-hydroxyethyl $\}-8$-hydroxy$2(1 \mathrm{H})$-quinolinone maleate. It is an ultra long acting beta adrenoceptor agonist. According to WHO, there are more than two hundred million people have moderate to severe COPD worldwide. It is in essential need of the theoretical properties. B3LYP/DFT/631-G basis set is used to calculate some physical properties of the target molecule with the help of Gaussian 09 software.

\section{Computational details}

The combination of quantum chemical calculation is very effective to understand the structure and behavior of the compound. The various analysis of the present study of the compound under investigation are carried out by DFT with three parameter hybrid [1,2] functional (B3) [3] for the exchange part and Lee YangParr [4] (LYP) correlation functional using 6-31G basis set. The Gaussian 09 package is used for this calculation [5].

\section{Result and Discussion}

\subsection{Optimized molecular structure}

The list of atoms for the target molecule is shown in Table 1. The optimized molecular structure is shown in Fig 1. The optimized bond length, bond angle and tetrahedral angles for Indacaterol molecule at both levels of theory are listed in Tables 2,3 and 4 respectively.

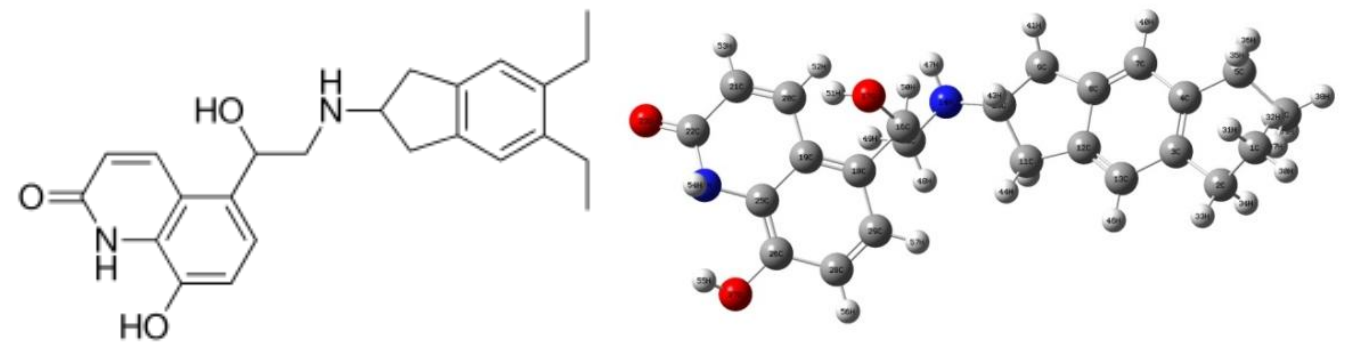

Fig 1: The 2D and 3D optimized molecular structure of Indacaterol

Table 1: The atom list of Indacaterol

\begin{tabular}{|l|l|l|l|l|l|l|l|}
\hline 1 & 2 & 3 & 4 & 5 & 6 & 7 & 8 \\
\hline $\mathbf{C}$ & $\mathbf{C}$ & $\mathbf{C}$ & $\mathbf{C}$ & $\mathbf{C}$ & $\mathbf{C}$ & $\mathbf{C}$ & $\mathbf{C}$ \\
\hline 9 & 10 & 11 & 12 & 13 & 14 & 15 & 16 \\
\hline $\mathbf{C}$ & $\mathbf{C}$ & $\mathbf{C}$ & $\mathbf{C}$ & $\mathbf{C}$ & $\mathbf{N}$ & $\mathbf{C}$ & $\mathbf{C}$ \\
\hline 17 & 18 & 19 & 20 & 21 & 22 & 23 & 24 \\
\hline $\mathbf{O}$ & $\mathbf{C}$ & $\mathbf{C}$ & $\mathbf{C}$ & $\mathbf{C}$ & $\mathbf{C}$ & $\mathbf{O}$ & $\mathbf{N}$ \\
\hline 25 & 26 & 27 & 28 & 29 & 30 & 31 & 32 \\
\hline $\mathbf{C}$ & $\mathbf{C}$ & $\mathbf{O}$ & $\mathbf{C}$ & $\mathbf{C}$ & $\mathbf{H}$ & $\mathbf{H}$ & $\mathbf{H}$ \\
\hline
\end{tabular}

National Conference on Current Advancements in Physics $3^{\text {rd }} \& 4^{\text {th }}$ February 2017 
Theoretical Study on Indacaterol by DFT Study

\begin{tabular}{|l|l|l|l|l|l|l|l|}
\hline 33 & 34 & 35 & 36 & 37 & 38 & 39 & 40 \\
\hline H & H & H & H & H & H & H & H \\
\hline 41 & 42 & 43 & 44 & 45 & 46 & 47 & 48 \\
\hline H & H & H & H & H & H & H & H \\
\hline 49 & 50 & 51 & 52 & 53 & 54 & 55 & 56 \\
\hline H & H & H & H & H & H & H & H \\
\hline 57 & & & & & & & \\
\hline H & & & & & & & \\
\hline
\end{tabular}

Table 2: Bond length of optimized Indacaterol molecule by 631-G calculation

\begin{tabular}{|c|c|c|c|c|c|c|c|}
\hline SI .no & Code & Bond & Bond distance $(\AA)$ & Sl .no & Code & Bond & Bond distance $(\AA)$ \\
\hline 1 & $\mathrm{R} 1$ & $\mathrm{R}(1,2)$ & 1.5464 & 31 & R31 & $\mathrm{R}(12,13)$ & 1.3937 \\
\hline 2 & R2 & $\mathrm{R}(1,30)$ & 1.0965 & 32 & R32 & $\mathrm{R}(13,46)$ & 1.0879 \\
\hline 3 & R3 & $\mathrm{R}(1,31)$ & 1.0959 & 33 & R33 & $\mathrm{R}(14,15)$ & 1.4659 \\
\hline 4 & R4 & $\mathrm{R}(1,32)$ & 1.0959 & 34 & R34 & $\mathrm{R}(14,47)$ & 1.0193 \\
\hline 5 & R5 & $\mathrm{R}(2,3)$ & 1.5207 & 35 & R35 & $\mathrm{R}(15,16)$ & 1.5351 \\
\hline 6 & R6 & $\mathrm{R}(2,33)$ & 1.0977 & 36 & R36 & $\mathrm{R}(15,48)$ & 1.0976 \\
\hline 7 & R7 & $\mathrm{R}(2,34)$ & 1.0964 & 37 & R37 & $\mathrm{R}(15,49)$ & 1.1044 \\
\hline 8 & R8 & $\mathrm{R}(3,4)$ & 1.421 & 38 & R38 & $\mathrm{R}(16,17)$ & 1.4839 \\
\hline 9 & R9 & $\mathrm{R}(3,13)$ & 1.4066 & 39 & R39 & $\mathrm{R}(16,18)$ & 1.5186 \\
\hline 10 & R10 & $\mathrm{R}(4,5)$ & 1.5265 & 40 & R40 & $\mathrm{R}(16,50)$ & 1.0957 \\
\hline 11 & R11 & $\mathrm{R}(4,7)$ & 1.407 & 41 & R41 & $\mathrm{R}(17,51)$ & 0.9789 \\
\hline 12 & R12 & $\mathrm{R}(5,6)$ & 1.5361 & 42 & R42 & $\mathrm{R}(18,19)$ & 1.4295 \\
\hline 13 & R13 & $\mathrm{R}(5,35)$ & 1.1009 & 43 & R43 & $\mathrm{R}(18,29)$ & 1.3943 \\
\hline 14 & R14 & $\mathrm{R}(5,36)$ & 1.099 & 44 & R44 & $\mathrm{R}(19,20)$ & 1.4487 \\
\hline 15 & R15 & $R(6,37)$ & 1.0961 & 45 & $\mathrm{R} 45$ & $\mathrm{R}(19,25)$ & 1.4144 \\
\hline 16 & R16 & $\mathrm{R}(6,38)$ & 1.0955 & 46 & R46 & $\mathrm{R}(20,21)$ & 1.3602 \\
\hline 17 & R17 & $R(6,39)$ & 1.0962 & 47 & R47 & $\mathrm{R}(20,52)$ & 1.0838 \\
\hline 18 & R18 & $\mathrm{R}(7,8)$ & 1.3952 & 48 & R48 & $\mathrm{R}(21,22)$ & 1.459 \\
\hline 19 & R19 & $\mathrm{R}(7,40)$ & 1.0845 & 49 & R49 & $\mathrm{R}(21,53)$ & 1.0826 \\
\hline 20 & $\mathrm{R} 20$ & $\mathrm{R}(8,9)$ & 1.5159 & 50 & R50 & $\mathrm{R}(22,23)$ & 1.2532 \\
\hline 21 & R21 & $\mathrm{R}(8,12)$ & 1.4036 & 51 & R51 & $\mathrm{R}(22,24)$ & 1.3985 \\
\hline 22 & R22 & $\mathrm{R}(9,10)$ & 1.572 & 52 & R52 & $\mathrm{R}(24,25)$ & 1.3831 \\
\hline 23 & R23 & $\mathrm{R}(9,41)$ & 1.0961 & 53 & R53 & $\mathrm{R}(24,54)$ & 1.0129 \\
\hline 24 & R24 & $\mathrm{R}(9,42)$ & 1.1021 & 54 & R54 & $\mathrm{R}(25,26)$ & 1.4105 \\
\hline 25 & R25 & $\mathrm{R}(10,11)$ & 1.5565 & 55 & R55 & $\mathrm{R}(26,27)$ & 1.3935 \\
\hline 26 & R26 & $R(10,14)$ & 1.4635 & 56 & R56 & $R(26,28)$ & 1.3851 \\
\hline 27 & $\mathrm{R} 27$ & $\mathrm{R}(10,43)$ & 1.0991 & 57 & R57 & $\mathrm{R}(27,55)$ & 0.9747 \\
\hline 28 & R28 & $\mathrm{R}(11,12)$ & 1.5145 & 58 & R58 & $\mathrm{R}(28,29)$ & 1.4079 \\
\hline 29 & R29 & $\mathrm{R}(11,44)$ & 1.0953 & 59 & R59 & $\mathrm{R}(28,56)$ & 1.0864 \\
\hline 30 & R30 & $\mathrm{R}(11,45)$ & 1.1022 & 60 & R60 & $\mathrm{R}(29,57)$ & 1.0826 \\
\hline
\end{tabular}

It is predicted that the longest and possibly weakest bonds are R1, R5, R10, R12, R20, R25, R28, R35 and R39 involving C-C bonds. The shortest and possibly strongest bonds are R41and R57 involving O-H bond.

Table 3: Bond angle of optimized Indacaterol molecule by 6-31G calculation

\begin{tabular}{|l|l|l|l|l|l|l|l|}
\hline Sl .no & Code & Bond & Bond angle $\left(^{\circ}\right)$ & Sl .no & Code & Bond & Bond angle $\left(^{\circ}\right)$ \\
\hline 1 & $\mathrm{~A} 1$ & $\mathrm{~A}(2,1,30)$ & 110.8945 & 55 & $\mathrm{~A} 55$ & $\mathrm{~A}(8,12,11)$ & 110.6888 \\
\hline 2 & $\mathrm{~A} 2$ & $\mathrm{~A}(2,1,31)$ & 110.6033 & 56 & $\mathrm{~A} 56$ & $\mathrm{~A}(8,12,13)$ & 119.7246 \\
\hline 3 & $\mathrm{~A} 3$ & $\mathrm{~A}(2,1,32)$ & 111.401 & 57 & $\mathrm{~A} 57$ & $\mathrm{~A}(11,12,13)$ & 129.585 \\
\hline 4 & $\mathrm{~A} 4$ & $\mathrm{~A}(30,1,31)$ & 108.1923 & 58 & $\mathrm{~A} 58$ & $\mathrm{~A}(3,13,12)$ & 120.7643 \\
\hline 5 & $\mathrm{~A} 5$ & $\mathrm{~A}(30,1,32)$ & 108.0415 & 59 & $\mathrm{~A} 59$ & $\mathrm{~A}(3,13,46)$ & 118.931 \\
\hline 6 & $\mathrm{~A} 6$ & $\mathrm{~A}(31,1,32)$ & 107.5726 & 60 & $\mathrm{~A} 60$ & $\mathrm{~A}(12,13,46)$ & 120.3035 \\
\hline 7 & $\mathrm{~A} 7$ & $\mathrm{~A}(1,2,3)$ & 113.1311 & 61 & $\mathrm{~A} 61$ & $\mathrm{~A}(10,14,15)$ & 116.6752 \\
\hline 8 & $\mathrm{~A} 8$ & $\mathrm{~A}(1,2,33)$ & 108.5676 & 62 & $\mathrm{~A} 62$ & $\mathrm{~A}(10,14,47)$ & 112.2483 \\
\hline 9 & $\mathrm{~A} 9$ & $\mathrm{~A}(1,2,34)$ & 109.1776 & 63 & $\mathrm{~A} 63$ & $\mathrm{~A}(15,14,47)$ & 110.1864 \\
\hline 10 & $\mathrm{~A} 10$ & $\mathrm{~A}(3,2,33)$ & 108.8281 & 64 & $\mathrm{~A} 64$ & $\mathrm{~A}(14,15,16)$ & 112.0089 \\
\hline 11 & $\mathrm{~A} 11$ & $\mathrm{~A}(3,2,34)$ & 110.7046 & 65 & $\mathrm{~A} 65$ & $\mathrm{~A}(14,15,48)$ & 108.8955 \\
\hline 12 & $\mathrm{~A} 12$ & $\mathrm{~A}(33,2,34)$ & 106.1729 & 66 & $\mathrm{~A} 66$ & $\mathrm{~A}(14,15,49)$ & 112.562 \\
\hline 13 & $\mathrm{~A} 13$ & $\mathrm{~A}(2,3,4)$ & 121.9505 & 67 & $\mathrm{~A} 67$ & $\mathrm{~A}(16,15,48)$ & 109.3157 \\
\hline 14 & $\mathrm{~A} 14$ & $\mathrm{~A}(2,3,13)$ & 118.4691 & 68 & $\mathrm{~A} 68$ & $\mathrm{~A}(16,15,49)$ & 106.933 \\
\hline
\end{tabular}


Theoretical Study on Indacaterol by DFT Study

\begin{tabular}{|c|c|c|c|c|c|c|c|}
\hline 15 & A15 & $\mathrm{A}(4,3,13)$ & 119.5664 & 69 & A69 & $\mathrm{A}(48,15,49)$ & 106.9627 \\
\hline 16 & A16 & $\mathrm{A}(3,4,5)$ & 120.006 & 70 & A70 & $\mathrm{A}(15,16,17)$ & 108.299 \\
\hline 17 & A17 & $\mathrm{A}(3,4,7)$ & 118.9815 & 71 & A71 & $\mathrm{A}(15,16,18)$ & 117.3853 \\
\hline 18 & A18 & $\mathrm{A}(5,4,7)$ & 121.0051 & 72 & A72 & $\mathrm{A}(15,16,50)$ & 106.6921 \\
\hline 19 & A19 & $\mathrm{A}(4,5,6)$ & 116.2799 & 73 & A73 & $\mathrm{A}(17,16,18)$ & 111.0973 \\
\hline 20 & A20 & $\mathrm{A}(4,5,35)$ & 108.8641 & 74 & A74 & $\mathrm{A}(17,16,50)$ & 103.928 \\
\hline 21 & A21 & $\mathrm{A}(4,5,36)$ & 109.0978 & 75 & A75 & $\mathrm{A}(18,16,50)$ & 108.5141 \\
\hline 22 & A22 & $\mathrm{A}(6,5,35)$ & 108.4027 & 76 & A76 & $\mathrm{A}(16,17,51)$ & 109.0385 \\
\hline 23 & A23 & $\mathrm{A}(6,5,36)$ & 108.4157 & 77 & A77 & $\mathrm{A}(16,18,19)$ & 118.851 \\
\hline 24 & A24 & $\mathrm{A}(35,5,36)$ & 105.2162 & 78 & A78 & $\mathrm{A}(16,18,29)$ & 122.1208 \\
\hline 25 & A25 & $\mathrm{A}(5,6,37)$ & 111.757 & 79 & A79 & $\mathrm{A}(19,18,29)$ & 119.0269 \\
\hline 26 & A26 & $\mathrm{A}(5,6,38)$ & 110.0556 & 80 & A80 & $\mathrm{A}(18,19,20)$ & 124.2265 \\
\hline 27 & A27 & $\mathrm{A}(5,6,39)$ & 111.605 & 81 & A81 & $\mathrm{A}(18,19,25)$ & 119.2921 \\
\hline 28 & A28 & $\mathrm{A}(37,6,38)$ & 107.5817 & 82 & A82 & $\mathrm{A}(20,19,25)$ & 116.481 \\
\hline 29 & A29 & $\mathrm{A}(37,6,39)$ & 107.9654 & 83 & A83 & $\mathrm{A}(19,20,21)$ & 121.6817 \\
\hline 30 & $\mathrm{~A} 30$ & $\mathrm{~A}(38,6,39)$ & 107.7003 & 84 & A84 & $\mathrm{A}(19,20,52)$ & 118.4848 \\
\hline 31 & A31 & $\mathrm{A}(4,7,8)$ & 120.7492 & 85 & A85 & $\mathrm{A}(21,20,52)$ & 119.7913 \\
\hline 32 & A32 & $\mathrm{A}(4,7,40)$ & 119.8168 & 86 & A86 & $\mathrm{A}(20,21,22)$ & 122.4013 \\
\hline 33 & A33 & $\mathrm{A}(8,7,40)$ & 119.4335 & 87 & A87 & $\mathrm{A}(20,21,53)$ & 121.7888 \\
\hline 34 & A34 & $\mathrm{A}(7,8,9)$ & 129.1896 & 88 & A88 & $\mathrm{A}(22,21,53)$ & 115.8073 \\
\hline 35 & A35 & $\mathrm{A}(7,8,12)$ & 120.2124 & 89 & A89 & $\mathrm{A}(21,22,23)$ & 125.0916 \\
\hline 36 & A36 & $\mathrm{A}(9,8,12)$ & 110.5972 & 90 & A90 & $\mathrm{A}(21,22,24)$ & 113.9689 \\
\hline 37 & A37 & $\mathrm{A}(8,9,10)$ & 102.9867 & 91 & A91 & $\mathrm{A}(23,22,24)$ & 120.938 \\
\hline 38 & A38 & $\mathrm{A}(8,9,41)$ & 113.4793 & 92 & A92 & $\mathrm{A}(22,24,25)$ & 125.2027 \\
\hline 39 & A39 & $\mathrm{A}(8,9,42)$ & 111.0859 & 93 & A93 & $\mathrm{A}(22,24,54)$ & 116.8442 \\
\hline 40 & $\mathrm{~A} 40$ & $\mathrm{~A}(10,9,41)$ & 112.7322 & 94 & A94 & $\mathrm{A}(25,24,54)$ & 117.9529 \\
\hline 41 & A41 & $\mathrm{A}(10,9,42)$ & 109.5127 & 95 & A95 & $\mathrm{A}(19,25,24)$ & 120.2448 \\
\hline 42 & $\mathrm{~A} 42$ & $\mathrm{~A}(41,9,42)$ & 107.0519 & 96 & A96 & $\mathrm{A}(19,25,26)$ & 120.2644 \\
\hline 43 & A43 & $\mathrm{A}(9,10,11)$ & 104.4006 & 97 & A97 & $\mathrm{A}(24,25,26)$ & 119.4906 \\
\hline 44 & A44 & $\mathrm{A}(9,10,14)$ & 117.2919 & 98 & A98 & $\mathrm{A}(25,26,27)$ & 114.9554 \\
\hline 45 & A45 & $\mathrm{A}(9,10,43)$ & 107.5829 & 99 & A99 & $\mathrm{A}(25,26,28)$ & 120.0758 \\
\hline 46 & $\mathrm{~A} 46$ & $\mathrm{~A}(11,10,14)$ & 111.9368 & 100 & A100 & $\mathrm{A}(27,26,28)$ & 124.9675 \\
\hline 47 & A47 & $\mathrm{A}(11,10,43)$ & 108.1677 & 101 & A101 & $\mathrm{A}(26,27,55)$ & 112.474 \\
\hline 48 & A48 & $\mathrm{A}(14,10,43)$ & 107.1117 & 102 & A102 & $\mathrm{A}(26,28,29)$ & 120.1199 \\
\hline 49 & A49 & $\mathrm{A}(10,11,12)$ & 103.4178 & 103 & A103 & $\mathrm{A}(26,28,56)$ & 120.075 \\
\hline 50 & A50 & $\mathrm{A}(10,11,44)$ & 111.9544 & 104 & A104 & $\mathrm{A}(29,28,56)$ & 119.8051 \\
\hline 51 & A51 & $\mathrm{A}(10,11,45)$ & 109.5582 & 105 & A105 & $\mathrm{A}(18,29,28)$ & 121.1875 \\
\hline 52 & A52 & $\mathrm{A}(12,11,44)$ & 114.1293 & 106 & A106 & $\mathrm{A}(18,29,57)$ & 118.9597 \\
\hline 53 & A53 & $\mathrm{A}(12,11,45)$ & 111.0329 & 107 & A107 & $\mathrm{A}(28,29,57)$ & 119.8227 \\
\hline 54 & A54 & $\mathrm{A}(44,11,45)$ & 106.7575 & & & & \\
\hline
\end{tabular}

From the Table 3, it is clear that A37, carbon atoms - 8,9,10 present in the five membered ring system shows the shortest angle while A57, carbons atoms 11,12,13 shows the largest angle.

From the tetrahedral values in Table 4, it is clear that the atoms of D1, D8, D19, D22, D23, D30, D31, D32, D33, D35, D43, D44, D45, D46, D53, D54, D55, D56, D87, D88, D89, D90, D131, D132, D135, D136, D137, D139, D140, D141, D142, D143, D144, D145, D146, D147, D148, D149, D150, D151, D152, D153, D154, D155, D156 and D157 lie in same plane.

Table: 4 Tetrahedral angle of optimized Indacaterol molecule by 631-G calculation

\begin{tabular}{|l|l|l|l|l|l|l|l|}
\hline Sl .no & Code & Bond & Bond angle $\left(^{\circ}\right)$ & Sl .no & Code & Bond & Bond angle $\left(^{\circ}\right)$ \\
\hline 1 & $\mathrm{D} 1$ & $\mathrm{D}(30,1,2,3)$ & 179.6524 & 81 & $\mathrm{D} 81$ & $\mathrm{D}(10,11,12,8)$ & 17.085 \\
\hline 2 & $\mathrm{D} 2$ & $\mathrm{D}(30,1,2,33)$ & 58.7415 & 82 & $\mathrm{D} 82$ & $\mathrm{D}(10,11,12,13)$ & -163.3913 \\
\hline 3 & $\mathrm{D} 3$ & $\mathrm{D}(30,1,2,34)$ & -56.5944 & 83 & $\mathrm{D} 83$ & $\mathrm{D}(44,11,12,8)$ & 138.9567 \\
\hline 4 & $\mathrm{D} 4$ & $\mathrm{D}(31,1,2,3)$ & 59.6169 & 84 & $\mathrm{D} 84$ & $\mathrm{D}(44,11,12,13)$ & -41.5196 \\
\hline 5 & $\mathrm{D} 5$ & $\mathrm{D}(31,1,2,33)$ & -61.294 & 85 & $\mathrm{D} 85$ & $\mathrm{D}(45,11,12,8)$ & -100.3317 \\
\hline 6 & $\mathrm{D} 6$ & $\mathrm{D}(31,1,2,34)$ & -176.6299 & 86 & $\mathrm{D} 86$ & $\mathrm{D}(45,11,12,13)$ & 79.192 \\
\hline 7 & $\mathrm{D} 7$ & $\mathrm{D}(32,1,2,3)$ & -59.9717 & 87 & $\mathrm{D} 87$ & $\mathrm{D}(8,12,13,3)$ & 0.0259 \\
\hline 8 & $\mathrm{D} 8$ & $\mathrm{D}(32,1,2,33)$ & 179.1174 & 88 & $\mathrm{D} 88$ & $\mathrm{D}(8,12,13,46)$ & -179.5661 \\
\hline 9 & $\mathrm{D} 9$ & $\mathrm{D}(32,1,2,34)$ & 63.7815 & 89 & $\mathrm{D} 89$ & $\mathrm{D}(11,12,13,3)$ & -179.4609 \\
\hline 10 & $\mathrm{D} 10$ & $\mathrm{D}(1,2,3,4)$ & 87.9956 & 90 & $\mathrm{D} 90$ & $\mathrm{D}(11,12,13,46)$ & 0.947 \\
\hline 11 & $\mathrm{D} 11$ & $\mathrm{D}(1,2,3,13)$ & -90.6379 & 91 & $\mathrm{D} 91$ & $\mathrm{D}(10,14,15,16)$ & 177.9432 \\
\hline 12 & $\mathrm{D} 12$ & $\mathrm{D}(33,2,3,4)$ & -151.2412 & 92 & $\mathrm{D} 92$ & $\mathrm{D}(10,14,15,48)$ & 56.9156 \\
\hline
\end{tabular}

National Conference on Current Advancements in Physics $3^{\text {rd }} \& 4^{\text {th }}$ February 2017

Department of Physics, St. John's College, Palayamkottai-627 002, Tamilnadu, India. DOI 10.9790/4861-17002037379 
Theoretical Study on Indacaterol by DFT Study

\begin{tabular}{|c|c|c|c|c|c|c|c|}
\hline 13 & D13 & $\mathrm{D}(33,2,3,13)$ & 30.1254 & 93 & D93 & $\mathrm{D}(10,14,15,49)$ & -61.5179 \\
\hline 14 & D14 & $\mathrm{D}(34,2,3,4)$ & -34.9152 & 94 & D94 & $\mathrm{D}(47,14,15,16)$ & -52.5504 \\
\hline 15 & D15 & $\mathrm{D}(34,2,3,13)$ & 146.4514 & 95 & D95 & $\mathrm{D}(47,14,15,48)$ & -173.578 \\
\hline 16 & D16 & $\mathrm{D}(2,3,4,5)$ & 2.6785 & 96 & D96 & $\mathrm{D}(47,14,15,49)$ & 67.9884 \\
\hline 17 & D17 & $\mathrm{D}(2,3,4,7)$ & -178.305 & 97 & D97 & $\mathrm{D}(14,15,16,17)$ & 55.4567 \\
\hline 18 & D18 & $\mathrm{D}(13,3,4,5)$ & -178.7027 & 98 & D98 & $\mathrm{D}(14,15,16,18)$ & -71.2904 \\
\hline 19 & D19 & $\mathrm{D}(13,3,4,7)$ & 0.3138 & 99 & D99 & $\mathrm{D}(14,15,16,50)$ & 166.793 \\
\hline 20 & D20 & $\mathrm{D}(2,3,13,12)$ & 178.3238 & 100 & D100 & $\mathrm{D}(48,15,16,17)$ & 176.2411 \\
\hline 21 & $\mathrm{D} 21$ & $\mathrm{D}(2,3,13,46)$ & -2.0786 & 101 & D101 & $\mathrm{D}(48,15,16,18)$ & 49.494 \\
\hline 22 & $\mathrm{D} 22$ & $\mathrm{D}(4,3,13,12)$ & -0.343 & 102 & D102 & $\mathrm{D}(48,15,16,50)$ & -72.4226 \\
\hline 23 & $\mathrm{D} 23$ & $\mathrm{D}(4,3,13,46)$ & 179.2545 & 103 & D103 & $\mathrm{D}(49,15,16,17)$ & -68.2993 \\
\hline 24 & D24 & $\mathrm{D}(3,4,5,6)$ & -177.6214 & 104 & D104 & $\mathrm{D}(49,15,16,18)$ & 164.9536 \\
\hline 25 & $\mathrm{D} 25$ & $\mathrm{D}(3,4,5,35)$ & 59.6414 & 105 & D105 & $\mathrm{D}(49,15,16,50)$ & 43.0369 \\
\hline 26 & D26 & $\mathrm{D}(3,4,5,36)$ & -54.6776 & 106 & D106 & $\mathrm{D}(15,16,17,51)$ & -106.512 \\
\hline 27 & $\mathrm{D} 27$ & $\mathrm{D}(7,4,5,6)$ & 3.3824 & 107 & D107 & $\mathrm{D}(18,16,17,51)$ & 23.7938 \\
\hline 28 & $\mathrm{D} 28$ & $\mathrm{D}(7,4,5,35)$ & -119.3548 & 108 & D108 & $\mathrm{D}(50,16,17,51)$ & 140.303 \\
\hline 29 & D29 & $\mathrm{D}(7,4,5,36)$ & 126.3262 & 109 & D109 & $\mathrm{D}(15,16,18,19)$ & -165.2877 \\
\hline 30 & D30 & $\mathrm{D}(3,4,7,8)$ & 0.0282 & 110 & D110 & $\mathrm{D}(15,16,18,29)$ & 14.286 \\
\hline 31 & D31 & $\mathrm{D}(3,4,7,40)$ & -179.7115 & 111 & D111 & $\mathrm{D}(17,16,18,19)$ & 69.3413 \\
\hline 32 & $\mathrm{D} 32$ & $\mathrm{D}(5,4,7,8)$ & 179.0345 & 112 & D112 & $\mathrm{D}(17,16,18,29)$ & -111.085 \\
\hline 33 & D33 & $\mathrm{D}(5,4,7,40)$ & -0.7052 & 113 & D113 & $\mathrm{D}(50,16,18,19)$ & -44.3166 \\
\hline 34 & D34 & $\mathrm{D}(4,5,6,37)$ & -61.4123 & 114 & D114 & $\mathrm{D}(50,16,18,29)$ & 135.2571 \\
\hline 35 & D35 & $\mathrm{D}(4,5,6,38)$ & 179.1202 & 115 & D115 & $\mathrm{D}(16,18,19,20)$ & -2.8007 \\
\hline 36 & D36 & $\mathrm{D}(4,5,6,39)$ & 59.6019 & 116 & D116 & $\mathrm{D}(16,18,19,25)$ & 177.4303 \\
\hline 37 & D37 & $\mathrm{D}(35,5,6,37)$ & 61.5658 & 117 & D117 & $\mathrm{D}(29,18,19,20)$ & 177.6122 \\
\hline 38 & D38 & $\mathrm{D}(35,5,6,38)$ & -57.9017 & 118 & D118 & $\mathrm{D}(29,18,19,25)$ & -2.1567 \\
\hline 39 & D39 & $\mathrm{D}(35,5,6,39)$ & -177.42 & 119 & D119 & $\mathrm{D}(16,18,29,28)$ & -177.9588 \\
\hline 40 & $\mathrm{D} 40$ & $\mathrm{D}(36,5,6,37)$ & 175.2888 & 120 & D120 & $\mathrm{D}(16,18,29,57)$ & 4.0389 \\
\hline 41 & $\mathrm{D} 41$ & $\mathrm{D}(36,5,6,38)$ & 55.8213 & 121 & D121 & $\mathrm{D}(19,18,29,28)$ & 1.6142 \\
\hline 42 & D42 & $\mathrm{D}(36,5,6,39)$ & -63.697 & 122 & D122 & $\mathrm{D}(19,18,29,57)$ & -176.3882 \\
\hline 43 & D43 & $\mathrm{D}(4,7,8,9)$ & 179.3098 & 123 & D123 & $\mathrm{D}(18,19,20,21)$ & 178.9093 \\
\hline 44 & D44 & $\mathrm{D}(4,7,8,12)$ & -0.3476 & 124 & D124 & $\mathrm{D}(18,19,20,52)$ & -3.4588 \\
\hline 45 & $\mathrm{D} 45$ & $\mathrm{D}(40,7,8,9)$ & -0.9495 & 125 & D125 & $\mathrm{D}(25,19,20,21)$ & -1.3158 \\
\hline 46 & $\mathrm{D} 46$ & $\mathrm{D}(40,7,8,12)$ & 179.3931 & 126 & D126 & $\mathrm{D}(25,19,20,52)$ & 176.3161 \\
\hline 47 & D47 & $\mathrm{D}(7,8,9,10)$ & 163.1545 & 127 & D127 & $\mathrm{D}(18,19,25,24)$ & -178.8929 \\
\hline 48 & $\mathrm{D} 48$ & $\mathrm{D}(7,8,9,41)$ & 40.977 & 128 & D128 & $\mathrm{D}(18,19,25,26)$ & 1.2363 \\
\hline 49 & D49 & $\mathrm{D}(7,8,9,42)$ & -79.6964 & 129 & D129 & $\mathrm{D}(20,19,25,24)$ & 1.3205 \\
\hline 50 & D50 & $\mathrm{D}(12,8,9,10)$ & -17.1618 & 130 & D130 & $\mathrm{D}(20,19,25,26)$ & -178.5502 \\
\hline 51 & D51 & $\mathrm{D}(12,8,9,41)$ & -139.3393 & 131 & D131 & $\mathrm{D}(19,20,21,22)$ & 0.2105 \\
\hline 52 & D52 & $\mathrm{D}(12,8,9,42)$ & 99.9873 & 132 & D132 & $\mathrm{D}(19,20,21,53)$ & 179.5953 \\
\hline 53 & D53 & $\mathrm{D}(7,8,12,11)$ & 179.8979 & 133 & D133 & $\mathrm{D}(52,20,21,22)$ & -177.3911 \\
\hline 54 & D54 & $\mathrm{D}(7,8,12,13)$ & 0.3206 & 134 & D134 & $\mathrm{D}(52,20,21,53)$ & 1.9938 \\
\hline 55 & D55 & $\mathrm{D}(9,8,12,11)$ & 0.1815 & 135 & D135 & $\mathrm{D}(20,21,22,23)$ & -179.5874 \\
\hline 56 & D56 & $\mathrm{D}(9,8,12,13)$ & -179.3957 & 136 & D136 & $\mathrm{D}(20,21,22,24)$ & 0.8649 \\
\hline 57 & D57 & $\mathrm{D}(8,9,10,11)$ & 26.7374 & 137 & D137 & $\mathrm{D}(53,21,22,23)$ & 0.9934 \\
\hline 58 & D58 & $\mathrm{D}(8,9,10,14)$ & 151.2438 & 138 & D138 & $\mathrm{D}(53,21,22,24)$ & -178.5543 \\
\hline 59 & D59 & $\mathrm{D}(8,9,10,43)$ & -88.0377 & 139 & D139 & $\mathrm{D}(21,22,24,25)$ & -0.8665 \\
\hline 60 & D60 & $\mathrm{D}(41,9,10,11)$ & 149.4167 & 140 & D140 & $\mathrm{D}(21,22,24,54)$ & 179.2401 \\
\hline 61 & D61 & $\mathrm{D}(41,9,10,14)$ & -86.0769 & 141 & D141 & $\mathrm{D}(23,22,24,25)$ & 179.565 \\
\hline 62 & D62 & $\mathrm{D}(41,9,10,43)$ & 34.6416 & 142 & D142 & $\mathrm{D}(23,22,24,54)$ & -0.3284 \\
\hline 63 & D63 & $\mathrm{D}(42,9,10,11)$ & -91.52 & 143 & D143 & $\mathrm{D}(22,24,25,19)$ & -0.2338 \\
\hline 64 & D64 & $\mathrm{D}(42,9,10,14)$ & 32.9864 & 144 & D144 & $\mathrm{D}(22,24,25,26)$ & 179.6379 \\
\hline 65 & D65 & $\mathrm{D}(42,9,10,43)$ & 153.7049 & 145 & D145 & $\mathrm{D}(54,24,25,19)$ & 179.6585 \\
\hline 66 & D66 & $\mathrm{D}(9,10,11,12)$ & -26.6923 & 146 & D146 & $\mathrm{D}(54,24,25,26)$ & -0.4697 \\
\hline 67 & D67 & $\mathrm{D}(9,10,11,44)$ & -150.0108 & 147 & D147 & $\mathrm{D}(19,25,26,27)$ & 179.8699 \\
\hline 68 & D68 & $\mathrm{D}(9,10,11,45)$ & 91.7531 & 148 & D148 & $\mathrm{D}(19,25,26,28)$ & 0.2725 \\
\hline 69 & D69 & $\mathrm{D}(14,10,11,12)$ & -154.5542 & 149 & D149 & $\mathrm{D}(24,25,26,27)$ & -0.0018 \\
\hline 70 & D70 & $\mathrm{D}(14,10,11,44)$ & 82.1273 & 150 & D150 & $\mathrm{D}(24,25,26,28)$ & -179.5992 \\
\hline 71 & D71 & $\mathrm{D}(14,10,11,45)$ & -36.1088 & 151 & D151 & $\mathrm{D}(25,26,27,55)$ & 179.9594 \\
\hline 72 & $\mathrm{D} 72$ & $\mathrm{D}(43,10,11,12)$ & 87.6703 & 152 & D152 & $\mathrm{D}(28,26,27,55)$ & -0.4657 \\
\hline 73 & D73 & $\mathrm{D}(43,10,11,44)$ & -35.6482 & 153 & D153 & $\mathrm{D}(25,26,28,29)$ & -0.8531 \\
\hline 74 & D74 & $\mathrm{D}(43,10,11,45)$ & -153.8843 & 154 & D154 & $\mathrm{D}(25,26,28,56)$ & 179.1297 \\
\hline
\end{tabular}


Theoretical Study on Indacaterol by DFT Study

\begin{tabular}{|l|l|l|l|l|l|l|l|}
\hline 75 & $\mathrm{D} 75$ & $\mathrm{D}(9,10,14,15)$ & 65.7398 & 155 & $\mathrm{D} 155$ & $\mathrm{D}(27,26,28,29)$ & 179.5923 \\
\hline 76 & $\mathrm{D} 76$ & $\mathrm{D}(9,10,14,47)$ & -62.7784 & 156 & $\mathrm{D} 156$ & $\mathrm{D}(27,26,28,56)$ & -0.4249 \\
\hline 77 & $\mathrm{D} 77$ & $\mathrm{D}(11,10,14,15)$ & -173.6299 & 157 & $\mathrm{D} 157$ & $\mathrm{D}(26,28,29,18)$ & -0.107 \\
\hline 78 & $\mathrm{D} 78$ & $\mathrm{D}(11,10,14,47)$ & 57.8519 & 158 & $\mathrm{D} 158$ & $\mathrm{D}(26,28,29,57)$ & 177.8783 \\
\hline 79 & $\mathrm{D} 79$ & $\mathrm{D}(43,10,14,15)$ & -55.2252 & 159 & $\mathrm{D} 159$ & $\mathrm{D}(56,28,29,18)$ & 179.9102 \\
\hline 80 & $\mathrm{D} 80$ & $\mathrm{D}(43,10,14,47)$ & 176.2566 & 160 & $\mathrm{D} 160$ & $\mathrm{D}(56,28,29,57)$ & -2.1045 \\
\hline
\end{tabular}

\subsection{Mullikan charges}

The bonding ability of a molecule depends on the electronic charge on the chelating atoms. The atomic charge values have been obtained by the Mullikan population analysis [6]. To confirm the reliability of the result, the Mullikan population analysis of the target molecule has been calculated using B3LYP/6-31G basis set. The Mullikan charges are given in Table 5. From the results, it is clear $22 \mathrm{C}$ is having very high value, since it attached with nitrogen atom and oxygen atom. Also $26 \mathrm{C}$ shows higher value due to the attachment of oxygen atom. All the hydrogen atoms were found to possess positive charge.

Table 5: Mullikan atomic charges of Indacaterol (Hartree)

\begin{tabular}{|c|c|c|c|c|c|c|c|c|}
\hline $\begin{array}{l}\text { Sl. } \\
\text { No }\end{array}$ & Atoms & $\begin{array}{c}\text { Atomic charges with } \\
\text { B3LYP }\end{array}$ & $\begin{array}{l}\text { Sl. } \\
\text { no }\end{array}$ & Atoms & $\begin{array}{c}\text { Atomic charges with } \\
\text { B3LYP }\end{array}$ & $\begin{array}{l}\text { Sl. } \\
\text { no }\end{array}$ & Atoms & $\begin{array}{c}\text { Atomic charges with } \\
\text { B3LYP }\end{array}$ \\
\hline 1 & $\mathrm{C}$ & -0.398892 & 20 & $\mathrm{C}$ & -0.092165 & 39 & $\mathrm{H}$ & 0.140695 \\
\hline 2 & $\mathrm{C}$ & -0.316669 & 21 & $\mathrm{C}$ & -0.163114 & 40 & $\mathrm{H}$ & 0.120137 \\
\hline 3 & $\mathrm{C}$ & 0.071831 & 22 & $\mathrm{C}$ & 0.506772 & 41 & $\mathrm{H}$ & 0.136876 \\
\hline 4 & $\mathrm{C}$ & 0.085315 & 23 & $\mathrm{O}$ & -0.470661 & 42 & $\mathrm{H}$ & 0.142955 \\
\hline 5 & $\mathrm{C}$ & -0.326371 & 24 & $\mathrm{~N}$ & -0.787431 & 43 & $\mathrm{H}$ & 0.138033 \\
\hline 6 & $\mathrm{C}$ & -0.410013 & 25 & $\mathrm{C}$ & 0.320134 & 44 & $\mathrm{H}$ & 0.142010 \\
\hline 7 & $\mathrm{C}$ & -0.195206 & 26 & $\mathrm{C}$ & 0.245138 & 45 & $\mathrm{H}$ & 0.139564 \\
\hline 8 & $\mathrm{C}$ & 0.068281 & 27 & $\mathrm{O}$ & -0.639857 & 46 & $\mathrm{H}$ & 0.116046 \\
\hline 9 & $\mathrm{C}$ & -0.317758 & 28 & $\mathrm{C}$ & -0.129374 & 47 & $\mathrm{H}$ & 0.275737 \\
\hline 10 & $\mathrm{C}$ & 0.023848 & 29 & $\mathrm{C}$ & -0.157536 & 48 & $\mathrm{H}$ & 0.138440 \\
\hline 11 & $\mathrm{C}$ & -0.313620 & 30 & $\mathrm{H}$ & 0.131596 & 49 & $\mathrm{H}$ & 0.131058 \\
\hline 12 & $\mathrm{C}$ & 0.082292 & 31 & $\mathrm{H}$ & 0.138839 & 50 & $\mathrm{H}$ & 0.148660 \\
\hline 13 & $\mathrm{C}$ & -0.199152 & 32 & $\mathrm{H}$ & 0.139910 & 51 & $\mathrm{H}$ & 0.363617 \\
\hline 14 & $\mathrm{~N}$ & -0.589553 & 33 & $\mathrm{H}$ & 0.132681 & 52 & $\mathrm{H}$ & 0.173499 \\
\hline 15 & $\mathrm{C}$ & -0.085881 & 34 & $\mathrm{H}$ & 0.132069 & 53 & $\mathrm{H}$ & 0.154086 \\
\hline 16 & $\mathrm{C}$ & 0.024189 & 35 & $\mathrm{H}$ & 0.145855 & 54 & $\mathrm{H}$ & 0.354835 \\
\hline 17 & $\mathrm{O}$ & -0.608652 & 36 & $\mathrm{H}$ & 0.145341 & 55 & $\mathrm{H}$ & 0.385164 \\
\hline 18 & $\mathrm{C}$ & 0.002986 & 37 & $\mathrm{H}$ & 0.139700 & 56 & $\mathrm{H}$ & 0.127041 \\
\hline 19 & $\mathrm{C}$ & 0.030804 & 38 & $\mathrm{H}$ & 0.131869 & 57 & $\mathrm{H}$ & 0.174004 \\
\hline
\end{tabular}

\subsection{Dipole moment, Quadrupole moment and various energies}

The dipole moment is the first derivative of the energy with respect to an applied field. It is a measure of the asymmetry in the molecular charge distribution and is given as a vector in three dimensions. The theoretical dipole moment (in Debye) is shown in Table 6. The total dipole moment is found to be 7.0504 Debye.

Table 6: The Dipole moment (in Debye) of Indacaterol

\begin{tabular}{|c|c|r|c|}
\hline \multicolumn{4}{|c|}{ B3LYP/6-31G } \\
\hline $\mathbf{X}$ & $\mathbf{Y}$ & $\mathbf{Z}$ & Total \\
\hline-6.0446 & 3.2090 & -1.6949 & 7.0504 \\
\hline
\end{tabular}

The predicted quadrupole moment for the target molecule is given in Table 7. It is clear that the molecule is slightly elongated along the XX axis in both levels of theory.

Table 7: The quadrupole moment of Indacaterol

\begin{tabular}{|c|c|c|}
\hline \multicolumn{3}{|c|}{ B3LYP/6-31G } \\
\hline $\mathbf{X X}$ & $\mathbf{Y Y}$ & $\mathbf{Z Z}$ \\
\hline-229.5692 & -155.9353 & -171.2368 \\
\hline
\end{tabular}

Thermo chemical analysis for the molecule is carried out at $298.15 \mathrm{~K}$ and 1 atmospheric pressure. The energies of thermal, constant volume heat capacity and entropy due to electronic, translational, rotational and vibrational energies are given in Table 8 . It is seen that vibrational energy accounts for almost all of the total energy. It is observed that the constant volume heat capacity due to vibrational energy is greater than all other. Similarly, the translational energy causes more entropy.

Table 8: Various energies of Indacaterol 


\begin{tabular}{|l|l|l|l|}
\hline \multirow{3}{*}{ Parameters } & \multicolumn{3}{|c|}{ B3LYP/6-31G } \\
\cline { 2 - 4 } & $\begin{array}{c}\mathrm{E} \\
\text { (Thermal) } \\
\text { KCal/Mol }\end{array}$ & $\begin{array}{c}\text { CV } \\
\text { Cal/Mol- } \\
\text { Kelvin }\end{array}$ & $\begin{array}{c}\text { S } \\
\text { Cal/Mol-Kelvin }\end{array}$ \\
\hline Total & 321.175 & 104.734 & 183.275 \\
\hline Electronic & 0.000 & 0.000 & 0.000 \\
\hline Translational & 0.889 & 2.981 & 43.792 \\
\hline Rotational & 0.889 & 2.981 & 37.013 \\
\hline Vibrational & 319.397 & 98.772 & 102.471 \\
\hline
\end{tabular}

The zero point correction, thermal correction to energy, thermal correction to enthalpy, thermal correction to Gibbs free energy, sum of electronic and zero point energy, sum of electronic and thermal energies, sum of electronic and thermal enthalpies and sum of electronic and thermal free energies are shown in Table 9. It is clear that the data are well correlated for both levels of theories.

Table 9: Thermo-chemistry of Indacaterol

\begin{tabular}{|l|l|}
\hline \multicolumn{1}{|c|}{ Parameters } & $\begin{array}{l}\text { B3LYP/6-31G } \\
\text { (Hartree/Particle) }\end{array}$ \\
\hline Zero-point correction & 0.484975 \\
\hline Thermal correction to Energy & 0.511825 \\
\hline Thermal correction to Enthalpy & 0.512769 \\
\hline Thermal correction to Gibbs Free Energy & 0.425689 \\
\hline Sum of electronic and zero-point Energies & -1265.789516 \\
\hline Sum of electronic and thermal Energies & -1265.762667 \\
\hline Sum of electronic and thermal Enthalpies & -1265.761723 \\
\hline Sum of electronic and thermal Free Energies & -1265.848803 \\
\hline
\end{tabular}

\subsection{Electrostatic Potential Map (ESP)}

ESP gives the electrostatic potential at location on a particular surface most commonly a surface of electron density to over all molecular size. In this target molecule (Fig 3) the colors near red represent large negative values [electron rich] while the colors near blue represent large positive values [electron poor] and the colors green and yellow represent intermediate values of the potential. [7] In this case, red, green, and yellow colors appeared near--OH, aromatic regions, -NH groups respectively.

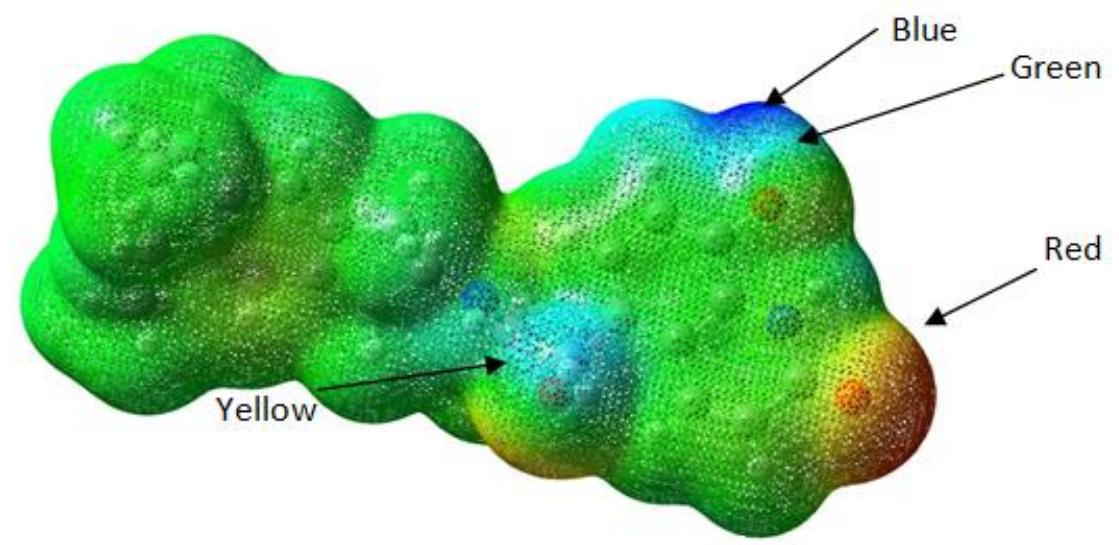

Fig 3: Electrostatic potential map of Indacaterol

\subsection{HOMO - LUMO and total energy}

From the Table 10, it is clear that the energy gap between HOMO and LUMO are high and hence the molecule is found to be stable. The total energy of the target molecule is found to be -1266.2744917 a.u.
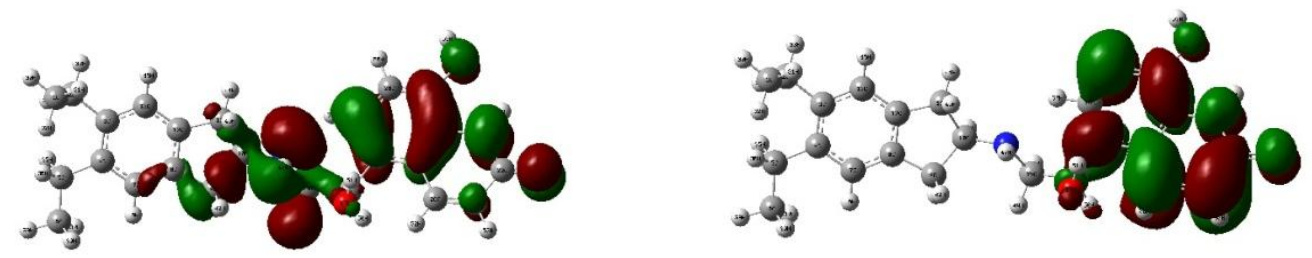

National Conference on Current Advancements in Physics $3^{\text {rd }} \& 4^{\text {th }}$ February 2017

Department of Physics, St. John's College, Palayamkottai-627 002, Tamilnadu, India. DOI 10.9790/4861-17002037379 


\section{a. $(\mathrm{HOMO})$}

b.(LUMO)

Fig 4: HOMO and LUMO of Indacaterol

Table 9: Physical parameters of Indacaterol

\begin{tabular}{|l|l|}
\hline & \multicolumn{1}{|c|}{$\mathbf{6 - 3 1 G}$} \\
\hline HOMO (a.u) & -0.21177 \\
\hline LUMO (a.u) & -0.05805 \\
\hline Energy gap (a.u) & -0.15372 \\
\hline Total energy (a.u) & -1266.2744917 \\
\hline
\end{tabular}

\section{Conclusion}

In this present study, computational methods are used to predict the molecular dynamics of indacaterol. The equilibrium geometry of Indacaterol molecule was analyzed at DFT/B3LYP/6-31G basis set. The HOMO, LUMO, energy gap, dipole moment and total energy etc., were calculated theoretically, The ESP has also been used to understand the activity of the molecule.

\section{References}

[1]. Dong Jun K, and Byung Tae C., Bulletin of Korean Chem. Soc., 24, 2003, 1641-1648.

[2]. Krishnamoorthy B, Kannan,Tangirala P, Saudi Pharmaceutical Journal, 20, 2012, 53-61.

[3]. Becke AD, J.ChemPhys, 98, 1993, 5648.

[4]. Lee C, Yang W X Par R.G, Phys Rev, 37B, 1988, 785.

[5]. Gaussian 09, Revision D.01, M. J. Frisch, G. W. Trucks, H. B. Schlegel, G. E. Scuseria, M. A. Robb, J. R. Cheeseman, G. Scalmani, V. Barone, B. Mennucci, G. A. Petersson, H. Nakatsuji, M. Caricato, X. Li, H. P. Hratchian, A. F. Izmaylov, J. Bloino, G. Zheng, J. L. Sonnenberg, M. Hada, M. Ehara, K. Toyota, R. Fukuda, J. Hasegawa, M. Ishida, T. Nakajima, Y.Honda, O. Kitao, H. Nakai, T. Vreven, J. A. Montgomery, Jr., J. E. Peralta, F. Ogliaro, M. Bearpark, J. J. Heyd, E. Brothers, K. N. Kudin, V. N. Staroverov, R. Kobayashi, J. Normand, K. Raghavachari, A. Rendell, J. C. Burant, S. S. Iyengar, J. Tomasi, M. Cossi, N. Rega, J. M. Millam, M. Klene, J. E. Knox, J. B. Cross, V. Bakken, C. Adamo, J. Jaramillo, R. Gomperts, R. E. Stratmann, O. Yazyev, A. J. Austin, R. Cammi, C. Pomelli, J. W. Ochterski, R. L. Martin, K. Morokuma, V. G. Zakrzewski, G. A. Voth, P. Salvador, J. J. Dannenberg, S. Dapprich, A. D. Daniels, Ö. Farkas, J. B. Foresman, J. V. Ortiz, J. Cioslowski, and D. J. Fox, Gaussian, Inc., Wallingford CT, 2009.

[6]. Mullikan, R. S, J.Chem. Phys., 23, 1955,1833.

[7]. Warren J Hehre, A Guide to Molecular Mechanics and Quantum Chemical Calculations Page 77. 